\title{
Awareness of Human Papillomavirus (HPV) Infection among Indigenous Males in North America and Oceania
}

Simran Bedi, ${ }^{1}$ Robert Strachan, ${ }^{1,2}$ Ingeborg Zehbe ${ }^{1,2}$

1. Biology Department, Lakehead University, Thunder Bay, Ontario, Canada

2. Probe Development and Biomarker Exploration, Thunder Bay Regional Research Institute, Thunder Bay, Ontario, Canada

Key Words: human papillomavirus; attitude; knowledge; belief; Indigenous; male; North America; Oceania

Correspondence to: Dr. Ingeborg Zehbe, Lakehead University - Biology Department, Thunder Bay, Ontario, P7B5E1, Canada;

Email: izehbe@lakeheadu.ca 


\begin{abstract}
We surveyed peer-reviewed literature on the awareness of human papillomavirus (HPV) infection among Indigenous males in North America and Oceania. Using keywords HPV plus male, men or boy, and ethnical filters such as Indigenous, Aboriginal or First Nations, we retrieved 47 articles based on titles of which we kept 14 after reading the abstracts. Reported HPV awareness was generally low in Indigenous males in North America with no peer-reviewed data from Oceania. The lower understanding by males compared to females was largely attributable to misconceptions about HPV-related diseases, their transmission, and prevention. Lack of awareness and concern toward the risk of contracting HPV infection in Indigenous males suggests an impediment in disseminating health information about this cancer-causing virus. Culturally sensitive education, with emphasis on Indigenous males, is needed to improve this group's HPV knowledge. Researchers should also engage meaningfully with Indigenous communities by building rapport to achieve a positive change in attitude.
\end{abstract}

\title{
Introduction
}

Worldwide, human papillomaviruses (HPVs) are the most common sexually transmitted infections (STIs) and therefore considered a global burden (Crow, 2012). Most are cleared by the immune response without clinical manifestation in the early stages of infection. Low-risk types 6 and 11 cause anogenital warts in men and women. High-risk HPVs, such as type 16, cause cervical cancer in some women with persistent infections, as well as cancers of the head, neck, and anogenital tract affecting both sexes (Crow, 2012; Gillison et al., 2014; Giuliano et al., 2011; 2015). To this end, oral HPV infection is a known cause of the steady increase in oropharyngeal squamous cell carcinoma (OPSSC) incidence, mostly among men, in several countries (review by Gillison et al., 2015 with references therein). While the risk of HPV infection decreases with age in women (Castle et al., 2005), men have the same risk of acquiring HPV throughout their lives (Giuliano et al., 2011). Optimal strategies for increasing HPV prevention in men remain to be established (Giuliano et al., 2011). In this regard, sexual health education is an important factor.

The scientific background why both girls and boys must be vaccinated against HPV to reduce the burden of HPV-related diseases has been elegantly elaborated and summarized in a recent endorsement by the German Standing Committee on Vaccination (Takla et al., 2018) entitled: "Background paper for the recommendation of HPV vaccination for boys in Germany". The running title Recommendation of HPV vaccination for boys was then further used to find other literature worldwide and yielded 80 hits. The most commonly covered topics included vaccination and males having sex with males, and a Canadian study emphasizing the collaboration with Media found that attitudes are becoming more positive toward HPV vaccination of males (Perez et al., 2016). The "scientific basis for global public health policies" has been emphasized in a Scandinavian study to eliminate HPV and its related diseases, particularly relating to gender neutrality (Lehtinen et al., 2019). Regrettably, the 2019 Human Papillomavirus and Related Diseases Report released by the International Agency for Research on Cancer is still too femalecentred, not even mentioning the male problem in the abstract (Bruni et al., 2019). Indeed, the importance of addressing HPV in males was concluded in a 2019 literature review (Lieblong et al., 2019) and a 2018 meta-analysis (Rodríguez-Álvarez et al., 2018). In a Turkish study of female and 
male teachers, a remarkable "knowledge deficiency" about HPV and vaccination was observed in men compared to women (Keten et al., 2019).

Here, we focus on Indigenous populations and HPV in males. Notably, in the Anishinaabek Cervical Cancer Screening Study, we showed that HPV-related health education is key to engaging Indigenous women in cervical screening (Zehbe et al., 2016; 2017). Moreover, the informants [all women] of our Anishinaabek partner communities underlined the importance to include men in all educational efforts so that they are aware that both men and women can contract HPV (Zehbe et al., 2017). HPV-related cancer incidence and mortality rates among Indigenous peoples in North America and Oceania are higher than in non-Indigenous peoples of their respective regions (Moore et al., 2015), making research into the barriers to HPV prevention faced by Indigenous peoples of immediate concern. Therefore, the main objective of this study was to survey the current literature on HPV knowledge among Indigenous males in North America and Oceania between 2010 and 2020 and deduce recommendations to increase HPV vaccination and decrease HPV burden in that population. These two regions were chosen as the main locales for the study because of their current public health focus on Indigenous communities and their similar colonial biographies.

\section{Methods}

\section{Literature search by titles and abstracts}

We searched by title using the PubMed database. The following format was used: *Keyword*, selected titles/total hits using a 10-year filter (2010-2020). Only peer-reviewed papers in English were selected. We started generally with *HPV male*, *HPV men* or *HPV boy* retrieving 2,381, 10,434 or 2,760 , respectively suggesting that the topic has received considerable coverage especially with regard to HPV vaccination. When using an additional filter related to ethnicity such as Aboriginal, Indigenous, Inuit, and others (Box 1) much fewer and overlapping hits were obtained $(n=292)$ amounting to a final total of 47 papers (Supplemental Data 1) of which we selected 14 based on reading the abstracts (Supplemental Data 2). In addition, we comment on Australia's vaccination program, which seems to successfully have included males, and especially Indigenous males, into the vaccination program (Hull et al., 2019). While this report was found through our PubMed database search, it seems to be a licensed work that was not peer-reviewed.

\section{Theoretical framework}

We used an inductive approach and Grounded Theory to create emerging themes from our literature search.

\section{Results}

Analysis of the methodologies of the selected papers indicated that most of the researchers (ten out of fourteen) adopted a qualitative approach (Dela Cruz et al., 2016; 2017; Devries et al., 2010; DiStefano et al., 2012; 2013; Henderson et al., 2018; Hodge, 2014; Itty et al., 2014; Nonzee et al., 2018; Soon et al., 2015); three were based on quantitative analysis (Dwojak et al., 2015; Gabster et al., 2019; Tom et al., 2016); and one had a mixed-methods approach (Hodge et al., 2011). 
Understanding attitudes, beliefs, and knowledge about HPV and men in Indigenous peoples is vital to addressing the increasing incidences of HPV-related cancers in their communities. After analyzing the articles, 4 themes relating to the HPV disease burden were identified that were consequences of beliefs and norms of the Indigenous community: (1) Low awareness and misconceptions about HPV; (2) Stigmatization of HPV-related diseases; (3) Increased HPV disease burden and low vaccination rates; and (4) Strategies for increasing HPV awareness.

\section{Low awareness and misconceptions about HPV}

There is a lack of accurate HPV knowledge among Indigenous peoples in North America and Oceania with misconceptions about HPV transmission, prevention and related diseases (DiStefano et al., 2012; Dwojak et al., 2015; Itty et al., 2014) and that males are not affected by HPV (DiStefano et al., 2012; Itty et al., 2014). Hodge et al. (2011) noted a significant difference between sexes and HPV risk status $(\mathrm{p}<0.01)$ as American Indian male students thought themselves to be at lower risk of contracting HPV infection when compared to female students. Adding to this, $43.5 \%$ of male participants believed that they were not at risk, compared to $8.8 \%$ of females (Hodge, 2014). Although an increase in HPV related knowledge is observed among females, males, especially fathers of male children, remain comparatively less educated, particularly in the Pacific Islander community (Nonzee et al., 2018). Furthermore, Hodge (2014) indicated that only $4.4 \%$ of males, when compared to $35.3 \%$ of females, were correct about the method of transmission of HPV. An assumption that men are just the "carriers" of HPV infection, and it only causes cervical cancer in women was also highlighted (Itty et al., 2014).

A study conducted by DiStefano et al. (2012) on Pacific Islanders indicated that talking about sexual health and intercourse between genders and generations is associated with taboo and a feeling of shame. In a combined knowledge and screening of head and neck cancer study with American Indians, it was noted that 59\% were aware of HPV (Dwojak et al., 2015). Of these, 87\% correctly believed that HPV is transmitted via sexual contact, but the authors found that there were considerable misconceptions about how this transmission happened. For instance, $22 \%$ believed that HPV is typically transmitted via coughing, $27 \%$ by eating something that may contain HPV and 54\% that HPV infection is brought about by poor hygiene (Dwojak et al., 2015). Along with this, the idea of hegemonic roles where men are expected to be aggressive and authoritative, whereas women have more passive behaviour when it comes to sexual activity is also widespread among Canadian Aboriginals residing in the urban Vancouver area and neighbouring rural areas (Devries et al., 2010). Indeed, low awareness and misconceptions of HPV transmission routes can lead to a higher risk of contracting HPV infection, which in turn can lead to more HPV-induced diseases.

\section{Stigmatization of HPV-related diseases}

Attitude and behaviour framed by the local culture towards sexuality and HPV may put women at higher risk of contracting HPV infection and developing cervical cancer. HPV is "taboo" and the responsibility of women, a view that is further compounded by an emphasis on targeting mostly women in different public HPV contexts (Hodge, 2014). Beliefs that stigmatized HPV to be only women's disease and fear from threatening their families' reputation often limited women from seeking medical advice on sexual health and getting screened (DiStefano et al., 2013). Both DiStefano et al. (2012) and Hodge (2014) pointed to the stigmatized nature of HPV in that the 
indigenous value system promotes modesty and privacy, thus limiting the open discussion of sexual health. It was stressed that rigorously maintaining participant confidentiality is of paramount importance to ease participants' fear of gossip due to the stigma associated with HPV (DiStefano et al., 2012; Dwojak et al., 2015). Until sexual shame is addressed by the community members, parents and healthcare providers, it is unlikely that indigenous males will seek information, let alone be interested in vaccination. On the other hand, the involvement of men in understanding and respecting women's health and body can cause remarkable improvement in cervical cancer rates and help alleviate the humiliation experienced when discussing sexual health (Henderson et al., 2018).

\section{Increased HPV disease burden and low vaccination rates}

Incidence of HPV-positive head and neck cancer is highest in white males, such as in the United States (Mahal et al., 2019; Stein et al., 2015) and Europe (Stein et al., 2015). An overall, current disbelief of being at low risk to contract HPV infection among Indigenous men may explain the practice of unprotected sex, increasing their risk of developing oropharyngeal, penile, and anal cancer in the future (Hodge, 2014). It is also not clear to most men, including Indigenous men, that partner-to-partner (male-to-female and vice versa) HPV infection may lead to cervical disease in women (Zehbe, unpublished observations). Moreover, strong religious beliefs were linked to preventing the use of condoms and, instead, limited the men to practice abstinence as the only means for protection (DiStefano et al., 2013). In the Comarca Ngäbe-Bugle population, 82\% of new STI infections reported were among males (Gabster et al., 2019). Administration of three doses of HPV vaccine to adolescents before they become sexually active has shown a considerable decrease in the incidence of HPV related infections and cancers. Despite this, the rates of HPV vaccination among children remain low, especially among North American Indigenous and Oceanic Indigenous populations (Dela Cruz et al., 2017; Hodge, 2014). Dela Cruz and colleagues (2016) also reported lower rates of HPV-vaccinated male children, where out of $69 \%$ of vaccinated children, only $12 \%$ were males. When compared between sexes, completion of the three-dose HPV vaccine is lower in males due to several barriers including lack of knowledge (Hodge et al., 2011), low perception of risk (Itty et al., 2014), lack of parents' consent (Nonzee et al., 2018) and lack of physician's recommendation (Soon et al., 2015). Moreover, parental belief that administration of the HPV vaccine might encourage sexual behaviour among adolescents was also repeatedly reported (Dela Cruz et al., 2017; Hodge et al., 2011). Using the same study population as Hodge (2014), Itty and colleagues (2014) reported sociodemographic factors such as the relative isolation of reserves, lower socioeconomic status, lack of opportunities available to Indigenous peoples, and the ethnocentrism of current HPV educational resources as factors limiting HPV knowledge transfer among American Indians. Adding to this, mistrust of the western health care system due to residential schools (Hodge et al., 2011) was identified by numerous sources as another significant barrier to HPV awareness (DiStefano et al., 2012; Dwojak et al., 2015; Hodge, 2014; Itty et al., 2014).

\section{Strategies for increasing HPV awareness}

From the reviewed studies we deduced various ways to increase HPV awareness among Indigenous men. There is a need to emphasize that both men and women are carriers of HPV and "responsible" for HPV disease transmission (Itty et al., 2014). Interestingly, according to 
DiStefano et al. (2012), men were believed to be at heightened risk of contracting HPV, due to risky behaviour, but the authors describe an adaptive cultural practice that could reduce such behaviours namely engaging people more with their community and palafa, a Tongan term that refers to bragging about fabricated sexual encounters to subvert peer pressure. Specific groups, such as adolescents (Gabster et al., 2019), smokers and alcohol users (Dwojak et al., 2015), as well as individuals from rural areas with little educational background (Devries et al., 2010) and inadequate financial opportunities (Dela Cruz et al., 2017), were identified as priority "at-risk" groups. Dwojak et al. (2015) stated that an important step in increasing awareness and decreasing HPV mortality rates is by increasing the frequency of community-based screening practices. Implementing a community-based approach to address the intergenerational communication gap has particularly been successful as it values the consent of community leaders before beginning any research (DiStefano et al., 2012; 2013; Henderson et al., 2018). Along with this, Hodge (2014) also pointed out the interest of Indigenous young adults in educating their siblings about sexual hygiene and STIs. While one study stressed that discussing sexual health matters within the family is a barrier (DiStefano et al., 2012), more recent findings highlighted the interest of older generations in educating young ones about sexual health and breaking the old norms (Henderson et al., 2018). However, there remain barriers to recommending the HPV vaccine to adolescents because of its perceived relation to sexual activity (Soon et al., 2015; Tom et al., 2016). Moreover, physicians were less likely to suggest vaccination to male compared to female children (Soon et al. 2015).

Providing and discussing educational brochures by health care professionals can increase the knowledge and acceptance of parents about the HPV vaccine (Del Cruz et al., 2017). The acceptability of the educational material can also be increased by making the brochure comprehensive in explaining both benefits and side effects of the HPV vaccine and include testimonies from health care professionals (Del Cruz et al., 2016). DiStefano et al. (2012) and Dwojak et al. (2015) also stressed the importance of maintaining participant confidentiality due to its paramount importance to ease participants' fear of gossip due to the shame associated with HPV. It was further recommended to distribute culturally appropriate resources with prevention programs for both sexes (Hodge, 2014), implement detailed STI education in public school curricula and relegate the stigma associated with HPV via intergenerational guidance from older siblings, family members and community members (DiStefano et al., 2012; 2013). Addressing the concerns faced by physicians to recommend HPV vaccination, like stocking and reimbursement issues, and the lack of an electronic record system for HPV vaccination status of patients could help boost the HPV vaccination rate in Indigenous adolescents.

\section{Comment}

\section{The Australian HPV vaccination program: a success story for uptake and HPV awareness?}

It seems that the Australian vaccination program remains a stand-alone success worldwide for Indigenous and non-Indigenous adolescent females and males. In their annual report, Hull and colleagues $(2019$, p. 1) stated that $79 \%$ and $77 \%$, respectively, of Indigenous girls and boys aged 15 years in 2017 completed three doses of the HPV vaccine, compared to $91 \%$ and $90 \%$ of nonIndigenous girls and boys, respectively. While the uptake in the Indigenous population is lower than that in the non-Indigenous, it is still remarkable compared to other countries with a similar colonial history. In an earlier report by the same team, it was noted that among Aboriginal women in the Northern Territory, the incidence of high-grade cervical abnormalities decreased by $41 \%$ in 
2010 alone (Dey et al., 2014, p. 136), due to the nationwide immunization and awareness campaign. The reported decrease in HPV infection among this population may be attributable to the manifold methods used during the vaccination campaign, such as voice-records of HPV resources in Aboriginal languages (Dey et al., 2014, p. 52), the incorporation of HPV into school curricula and outside the school setting, such as in community clinics and shopping centres (Dey et al., 2014, p. 63), promotion through health staff in remote areas (Dey et al., 2014, p. 63), and making written resources catered to Indigenous people available in the communities (Dey et al., 2014, p. 64). It was also noted that the "girls paved the way for the boys" and by implementing a gender-neutral and nation-wide vaccination program (Dey et al., 2014, p. 72), HPV vaccine acceptability may have increased both in Indigenous and non-Indigenous males. Although the authors do state that maintaining culturally appropriate materials was an ongoing challenge, due to the diversity of Aboriginal cultures in Australia (Dey et al., 2014, p. 65), further success came from increasing the frequency of educational campaigns and having a clear message that HPV is an STI that affects everyone (Dey et al., 2014, p. 73).

\section{Limitations}

Although HPV and males has been assessed in the Indigenous Oceanic male population, this review was unable to identify any peer-reviewed literature on the topic. Predominantly electronic searches were conducted and because titles and abstracts were initially screened it is possible that some studies with a focus on Indigenous males, but without any indication of this in the title or abstract, were overlooked. This review also only assessed those reports presented in English. Risk of bias across publications was addressed only by Devries et al. (2010): "We also recognise that our own positions, both culturally and socially, have shaped how participants communicate with us and how we interpret those communications." Indeed, recruitment strategies used such as convenience, word of mouth, and purposive sampling may indicate bias. We could not find any study comparing Indigenous and non-Indigenous males regarding HPV awareness or one that dealt with Indigenous males and HPV alone.

\section{Conclusions}

The reviewed literature suggests that HPV awareness among Indigenous males is lower than in Indigenous females, and this may be attributed in part to the gendered attribution of HPV as a "female problem", as well as the lack of targeted and culturally shaped educational initiatives focusing on males. However, this gap in HPV knowledge suggests upstream, systemic issues, in that the current HPV campaigns simply are not as effective at reaching Indigenous males as they are at reaching Indigenous females (with the exception of HPV vaccination in Australia). The lower knowledge of HPV observed among Indigenous males reaffirms the need for policy shifts in contemporary Indigenous health care education and delivery at the government and organizational levels to seriously address this issue. As seen in Dey et al.'s (2014) analysis, a wide array of methods including educational materials, deployed in culturally acceptable and public contexts, and engagement in rural communities through health staff, helped to decrease the elevated incidence of HPV-related diseases observed for females in this community. Indeed, rather than focusing on this group alone, it is also necessary to identify males as proportionally 
responsible for the prevention of HPV and subsequent disease burden. As more countries begin to authorize HPV vaccination of males it is also prudent to provide a proportional amount of education targeted at both sexes, specifically, and in culturally sensitive and respectful manners to increase awareness and maximize the efficacy of HPV prevention mandates of all ethnicities living in a country.

\section{Acknowledgments}

This work was funded by the Canadian Institutes of Health Research (CIHR) with IZ being the nominated Principal Investigator (CIHR \# MOP-119344).

\section{Ethics Approval}

Because this study did not engage in data collection or interact with any human or animal subjects, ethics approval was not sought.

\section{References}

Bruni L, Albero G, Serrano B, Mena M, Gómez D, Muñoz J, Bosch FX, de Sanjosé S. ICO/IARC Information Centre on HPV and Cancer (HPV Information Centre). Human Papillomavirus and Related Diseases in the World. Summary Report 17 June 2019. [8 April 2020].

Castle PE, Schiffman M, Herrero R, et al. A prospective study of age trends in cervical human papillomavirus acquisition and persistence in Guanacaste, Costa Rica. J Infect Dis 2005;191(11):1808-1816. DOI:10.1086/428779.

Crow JM. HPV: The global burden. Nat 2012;488(7413):S2-S3. DOI:10.1038/488S2a. Dela Cruz MRI, Tsark JAU, Chen JJ, Albright CL, Braun KL. Human Papillomavirus (HPV) Vaccination Motivators, Barriers, and Brochure Preferences Among Parents in Multicultural Hawai'i: a Qualitative Study. J Cancer Educ 2017;32(3):613-621. DOI:10.1007/s13187-0161009-2.

Dela Cruz MR, Tsark JA, Soon R, Albright CL, Braun KL. Insights in Public Health: Community Involvement in Developing a Human Papillomavirus (HPV) Vaccine Brochure Made for Parents in Hawai'i. J Med Public Health 2016;75(7):203-207.

Devries KM, Free C. 'I told him not to use condoms': masculinities, femininities and sexual health of Aboriginal Canadian young people. Sociol Health Illn 2010;32(6):827-842.

DOI:10.1111/j.1467-9566.2010.01242.x.

Dey A, Knox S, Brotherton J, et al. Evaluation of the National Papillomavirus Vaccination Program FINAL REPORT. National Centre for Immunisation Research \& Surveillance. NCIRS. 28 August 2014 [8 April 2020]. 
DiStefano AS, Hui B, Barrera-Ng A, et al. Contextualization of HIV and HPV risk and prevention among Pacific Islander young adults in Southern California. Soc Sci Med 2012;75(4):699-708. DOI:10.1016/j.socscimed.2012.04.011.

DiStefano A, Quitugua L, Hui B, Barrera-Ng A, Peters R, Vunileva I, et al. A community-based participatory research study of HIV and HPV vulnerabilities and prevention in two Pacific Islander communities: ethical challenges and solutions. J Empir Res Hum Res Ethics 2013;8(1):68-78. DOI:10.1525/jer.2013.8.1.68

Dwojak S, Deschler D, Sargent M, Emerick K, Guadagnolo BA, Petereit D. Knowledge and screening of head and neck cancer among American Indians in South Dakota. Am J Public Health 2015;105(6):1155-1160. DOI:10.2105/AJPH.2014.302177.

Gabster A, Pascale JM, Cislaghi B, Francis SC, Weiss HA, Martinez A, et al. High Prevalence of Sexually Transmitted Infections, and High-Risk Sexual Behaviors Among Indigenous Adolescents of the Comarca Ngäbe-Buglé, Panama [published correction appears in Sex Transm Dis. 2020 Feb;47(2):e8]. Sex Transm Dis 2019;46(12):780-787.

DOI:10.1097/OLQ.0000000000001070.

Gillison ML, Castellsagué X, Chaturvedi A, Goodman MT, Snijders P, Tommasino M, et al. Eurogin Roadmap: comparative epidemiology of HPV infection and associated cancers of the head and neck and cervix. Int J Cancer 2014;134(3):497-507. DOI:10.1002/ijc.28201.

Gillison ML, Chaturvedi AK, Anderson WF, Fakhry C. Epidemiology of Human Papillomavirus-Positive Head and Neck Squamous Cell Carcinoma. J Clin Oncol 2015;33(29):3235-3242. DOI:10.1200/JCO.2015.61.6995.

Giuliano AR, Lee JH, Fulp W, Villa LL, Lazcano E, Papenfuss MR, et al. Incidence and clearance of genital human papillomavirus infection in men (HIM): a cohort study [published correction appears in The Lancet. 2011 Jun 11;377(9782):2006]. Lancet 2011;377(9769):932940. DOI:10.1016/S0140-6736(10)62342-2.

Giuliano AR, Nyitray AG, Kreimer AR, Pierce Campbell CM, Goodman MT, Sudenga SL, et al. EUROGIN 2014 roadmap: differences in human papillomavirus infection natural history, transmission and human papillomavirus-related cancer incidence by gender and anatomic site of infection. Int J Cancer 2015;136(12):2752-2760. DOI:10.1002/ijc. 29082.

Henderson RI, Shea-Budgell M, Healy C, Letendre A, Bill L, Healy B, et al. First nations people's perspectives on barriers and supports for enhancing HPV vaccination: Foundations for sustainable, community-driven strategies. Gynecol Oncol 2018;149(1):93-100.

DOI:10.1016/j.ygyno.2017.12.024.

Hodge FS, Itty T, Cardoza B, Samuel-Nakamura C. HPV vaccine readiness among American Indian college students. Ethn Dis 2011;21(4):415-420. 
Hodge FS. American Indian Male College Students Perception and Knowledge of Human Papillomavirus (HPV). J Vaccines Vaccin 2014;5:222. DOI:10.4172/2157-7560.1000222.

Hull B, Hendry A, Dey A, Brotherton J, Macartney K, Beard F. Annual Immunisation Coverage Report 2017. Commun Dis Intell (2018) 2019;43:10.33321/cdi.2019.43.47. Published 2019 Nov 18. DOI:10.33321/cdi.2019.43.47.

Itty TL, Hodge FS, Poitra C, Cardoza B. HPV awareness among American Indian young adults: implications for health education. J Cult Divers 2014;21(4):123-129.

Keten HS, Ucer H, Dalgaci AF, Isik O, Ercan Ö, Guvenc N. Knowledge, Attitude, and Behavior of Teachers Regarding HPV (Human Papillomavirus) and Vaccination. J Cancer Educ 2019;16:1-7. DOI:10.1007/s13187-019-01668-2.

Lieblong BJ, Montgomery BEE, Su LJ, Nakagawa M. Natural history of human papillomavirus and vaccinations in men: A literature review. Health Sci Rep. 2019;2(5):e118.

DOI:10.1002/hsr2.118.

Lehtinen M, Baussano I, Paavonen J, Vänskä S, Dillner J. Eradication of human papillomavirus and elimination of HPV-related diseases-scientific basis for global public health policies. Expert Rev Vaccines 2019;18(2):153-160. DOI:10.1080/14760584.2019.1568876.

Mahal BA, Catalano PJ, Haddad RI, Hanna GJ, Kass JI, Schoenfeld JD, et al. Incidence and Demographic Burden of HPV-Associated Oropharyngeal Head and Neck Cancers in the United States. Cancer Epidemiol Biomarkers Prev 2019;28(10):1660-1667. DOI:10.1158/10559965.EPI-19-0038.

Moore SP, Antoni S, Colquhoun A, Healy B, Ellison-Loschmann L, Potter JD, et al. Cancer incidence in indigenous people in Australia, New Zealand, Canada, and the USA: a comparative population-based study. Lancet Oncol 2015;16(15):1483-1492. DOI:10.1016/S14702045(15)00232-6.

Nonzee NJ, Baldwin SB, Cui Y, Singhal R. Disparities in parental human papillomavirus (HPV) vaccine awareness and uptake among adolescents. Vaccine 2018;36(10):1243-1247.

DOI:10.1016/j.vaccine.2017.08.046.

Perez S, Fedoruk C, Shapiro GK, Rosberger Z. Giving Boys a Shot: The HPV Vaccine's Portrayal in Canadian Newspapers. Health Commun 2016;31(12):1527-1538. DOI:10.1080/10410236.2015.1089466.

Rodríguez-Álvarez MI, Gómez-Urquiza JL, Husein-El Ahmed H, Albendín-García L, GómezSalgado J, Cañadas-De la Fuente GA. Prevalence and Risk Factors of Human Papillomavirus in Male Patients: A Systematic Review and Meta-Analysis. Int J Environ Res Public Health 2018;15(10):2210. doi:10.3390/ijerph15102210. 
Soon R, Cruz MR, Tsark JU, Chen JJ, Braun KL. A Survey of Physicians' Attitudes and Practices about the Human Papillomavirus (HPV) Vaccine in Hawai 'i. Hawaii J Med Public Health 2015;74(7):234-241.

Stein AP, Saha S, Kraninger JL, Swick AD, Yu M, Lambertg PF, et al. Prevalence of Human Papillomavirus in Oropharyngeal Cancer: A Systematic Review. Cancer J 2015;21(3):138-146. DOI:10.1097/PPO.0000000000000115.

Takla A, Wiese-Posselt M, Harder T, Meerpohl JJ, Röbl-Mathieu M, Terhardt M, et al. Background paper for the recommendation of HPV vaccination for boys in Germany. Bundesgesundheitsblatt Gesundheitsforschung Gesundheitsschutz 2018;61(9):11701186. doi:10.1007/s00103-018-2791-2.

Tom A, Robinett H, Buenconsejo-Lum L, Soon R, Hamilton M, Francisco-Natanauan P, et al. Promoting and Providing HPV Vaccination in Hawaii: Barriers Faced by Health Providers. J Commun Health 2016;41(5):1069-1077. DOI:10.1007/s10900-016-0191-9.

Zehbe I, Wakewich P, Wood B, Sameshima P, Banning Y, Little J. Engaging Canadian First Nations Women in Cervical Screening through Education. Int J Health Promotion Educ 2016;54(5):255-264. DOI:10.1080/14635240.2016.1169942.

Zehbe I, Wakewich P, King AD, Morrisseau K, Tuck C. Self-administered versus providerdirected sampling in the Anishinaabek Cervical Cancer Screening Study (ACCSS): a qualitative investigation with Canadian First Nations women. BMJ Open 2017;7(8):e017384. DOI:10.1136/bmjopen-2017-017384. 